\title{
NOTES
}

\section{Melting-Recrystallization of Block Copolymer Crystals in Confined Environments}

\author{
Jun-Ting $\mathrm{XU},{ }^{\dagger}$ Yan-Qin ZHAO, Guo-Dong LIANG, and Zhi-Qiang FAN \\ Department of Polymer Science \& Engineering, Zhejiang University, Hangzhou 310027, China
}

(Received May 18, 2004; Accepted September 27, 2004; Published January 15, 2005)

KEY WORDS Crystallization / Block Copolymer / Melting /

[DOI 10.1295/polymj.37.43]

Recently crystallization of block copolymers in confined domains has been extensively studied. ${ }^{1-11}$ Crystallization in confined environments is usually initiated by homogeneous nucleation at large supercooling. ${ }^{2,10,12}$ Thus the crystals formed are highly imperfect and have lower crystallinity, ${ }^{5,11}$ compared with the crystals formed under normal crystallization conditions. As a result, the possibility of reorganization of these less perfect crystals into more ordered crystals is of great interest. Such reorganization can be achieved by melting-recrystallization or solid-solid transformation. Rottele et al. investigated melting behavior of strongly segregated poly(butadiene)- $b$-poly(ethylene oxide) (PB- $b$-PEO) block copolymers with spherical PEO domains. ${ }^{13}$ They observed that PEO crystals with different degrees of perfection could be produced when crystallization of PEO takes place in the confined spherical domains and the less perfect crystals transform into more perfect ones by reorganization in the solid state, but reorganization by melting-recrystallization is prohibited. ${ }^{13}$ Chen also observed coalescence of different crystalline domains after annealing the confined domains of PB- $b$-PEO block copolymer. ${ }^{14}$ However, reorganization of confined crystals via melting-recrystallization has not been reported so far. Confined crystallization behavior of semicrystalline block copolymers is strongly influenced by segregation strength of the two blocks and the morphology in the ordered melt. ${ }^{9,11}$ The effects of these two factors on reorganization of the block copolymer crystals have not been reported. In the present work, an oxyethylene/oxybutylene, generally believed to be a weakly segregated block copolymer, was blended with amorphous poly(oxybutylene) homopolymer. Two blends with $\phi_{\mathrm{B}}=0.76$ (cylinder morphology) and $\phi_{\mathrm{B}}=0.83$ (sphere morphology), respectively, were prepared and both exhibited confined crystallization behavior. ${ }^{11}$ Reorganization of the PEO crystals in confined domains of different shapes was studied with differential scanning calorimetry (DSC) and synchrotron small angle X-ray scattering (SAXS) at different heating rates.

\section{EXPERIMENTAL}

\section{Preparation of the Blends}

The blends were prepared by mixing an oxyethylene/oxybutylene block copolymer having a narrow molecular weight distribution, $\mathrm{E}_{155} \mathrm{~B}_{76}$ with different amounts of amorphous poly(oxybutylene). The subscript numbers of the notation denote the polymerization degrees of the oxyethylene and oxybutylene units. The blends were designated as E155-76 and E155-83, where the last two numbers are the percentages of the amorphous component in the blend. E155-76 and E155-83 have cylinder and sphere morphology in the molten state $\left(70^{\circ} \mathrm{C}\right)$, respectively, as determined by small angle X-ray scattering (SAXS). ${ }^{11}$

\section{DSC Experiments}

DSC experiments were carried out on a Pyris-1 instrument. The samples were first held at $70^{\circ} \mathrm{C}$ for 5 min and then cooled to $-50^{\circ} \mathrm{C}$ at a rate of $10^{\circ} \mathrm{C} /$ min. The melting behavior of the blends was investigated at prescribed heating rates.

\section{SAXS}

Time-resolved SAXS experiments were conducted at beamlines 8.2 of the SRS at the Daresbury, Warrington, U.K. The samples were $3.5 \mathrm{~m}$ far from the detector. The data were collected in $10 \mathrm{~s}$ frames separated by a waiting-time of $10 \mu \mathrm{s}$. Details of the instrument and data processing are described elsewhere. $^{11}$

${ }^{\dagger}$ To whom correspondence should be addressed (Fax: +86-571-87952400, E-mail: xujt@zju.edu.cn). 


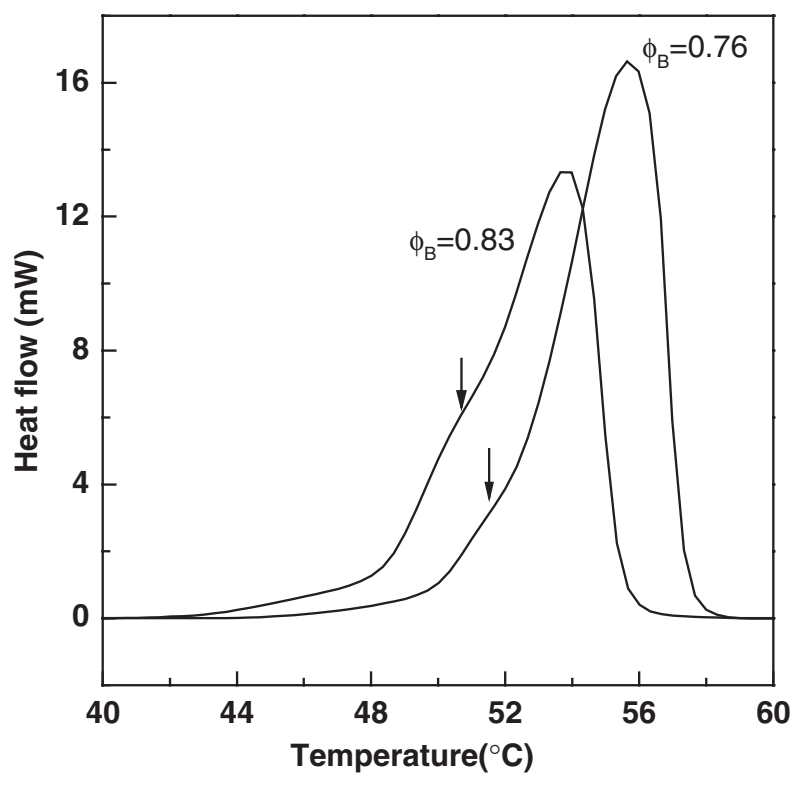

Figure 1. DSC melting traces of E155-76 and E155-83 at a heating rate of $10^{\circ} \mathrm{C} / \mathrm{min}$ after non-isothermal crystallization. The cooling rate in the non-isothermal crystallization is $10^{\circ} \mathrm{C} /$ $\min$. The arrows indicate shoulder melting peaks at lower temperatures.

\section{RESULTS AND DISCUSSION}

Figure 1 shows the DSC melting traces of E155-76 and $\mathrm{E} 155-83$ at a heating rate of $10^{\circ} \mathrm{C} / \mathrm{min}$ after nonisothermal crystallization. The cooling rate in non-isothermal crystallization was $10^{\circ} \mathrm{C} / \mathrm{min}$. Both blends show major melting peaks together with shoulder peaks at lower temperature. The appearance of double melting peaks at a fast heating rate indicates the existence of PEO crystals with different degrees of perfection in both blends. As revealed in our previous work, confined crystallization occurs in both blends at such a cooling rate. ${ }^{10}$ To examine the ability of reorganization of the PEO crystals formed in these two blends, a slower heating rate of $0.5^{\circ} \mathrm{C} / \mathrm{min}$ was applied to the blends after cooled at a rate of $10^{\circ} \mathrm{C} / \mathrm{min}$ and the melting traces are illustrated in Figure 2. There was nearly no change in the shape of DSC trace for E155-83 and the shoulder melting peak at lower temperature was observed as well at such a slow heating rate. In contrast, the DSC trace of E155-76 is quite different at the heating rate of $0.5^{\circ} \mathrm{C} / \mathrm{min}$. The major melting peak becomes weaker and a third melting peak appears beyond the original high temperature peak. Because of the extremely slow heating rate, any possible reorganization during melting process will be probed by DSC. The unchanged DSC profile at the slow heating rate shows that no reorganization occurs for E155-83 in spite of less perfect PEO crystals. Nevertheless, the appearance of the third melting peak at higher temperature indicates that reorganiza-

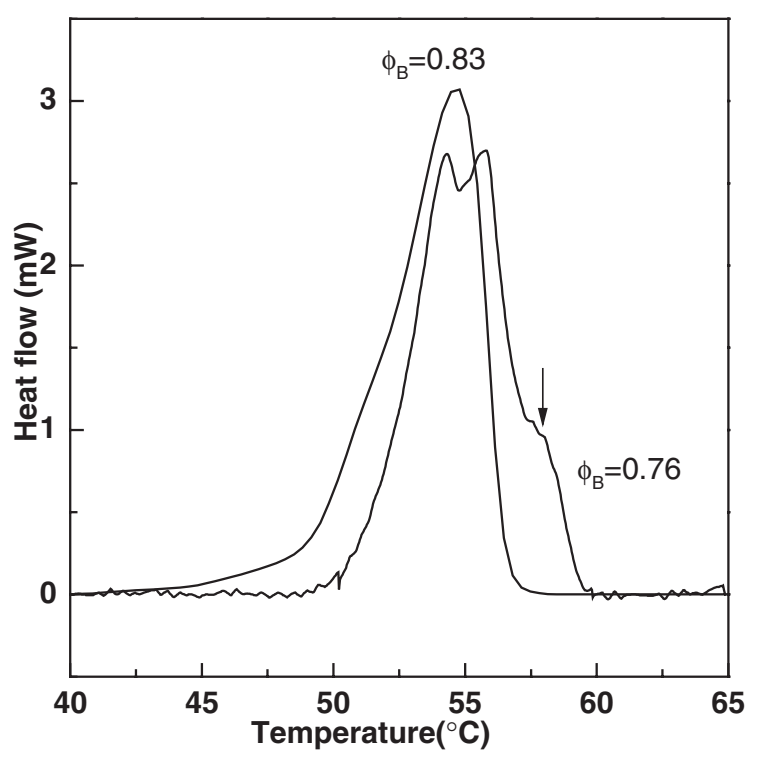

Figure 2. DSC melting traces of E155-76 and E155-83 at a heating rate of $0.5^{\circ} \mathrm{C} / \mathrm{min}$ after non-isothermal crystallization. The cooling rate in the non-isothermal crystallization is $10^{\circ} \mathrm{C} /$ min. The arrow indicates a newly appearing melting peak in E155-76.

tion takes place during melting of E155-76 at a slow heating rate.

To verify the results by DSC, time-resolved SAXS was used to monitor the melting of E155-76 and E155-83. Both samples undergo non-isothermal crystallization at a cooling rate of $10^{\circ} \mathrm{C} / \mathrm{min}$ from 70 to $-50^{\circ} \mathrm{C}$. Our previous results showed that in this process both samples exhibit confined crystallization behavior, since the position of the first-order SAXS peak, $q^{*}$, shows a continuous change instead of a stepwise change after the PEO block crystallizes. ${ }^{10}$ The samples were heated to $40^{\circ} \mathrm{C}$ at rate of $10^{\circ} \mathrm{C} / \mathrm{min}$ and heated from 40 to $65^{\circ} \mathrm{C}$ at a rate of $0.5^{\circ} \mathrm{C} / \mathrm{min}$. The changes of $q^{*}$ in the second heating step are shown in Figure 3. The sample E155-76 and E15583 have cylinder and sphere morphologies, respectively, and the melting characteristics of confined cylinder and sphere morphologies are different. ${ }^{11}$ Thus we should compare the present results with standard melting characteristics of confined cylinder and sphere morphologies. For E155-83 there is no appreciable change of $q^{*}$ during melting, but $q^{*}$ starts to change linearly with temperature after the crystals melts, since the liquid is easier to deform than the solid. Such phenomenon is exactly the same as the melting characteristic of confined sphere morphology. ${ }^{11}$ Thus the PEO crystals in E155-83 are still confined at such slow heating rate and no re-organization takes place. During melting of the crystals confined in cylinder domains, stepwise increase in the $q^{*}$ is observed, since the liquid and solid domains have different sizes at 


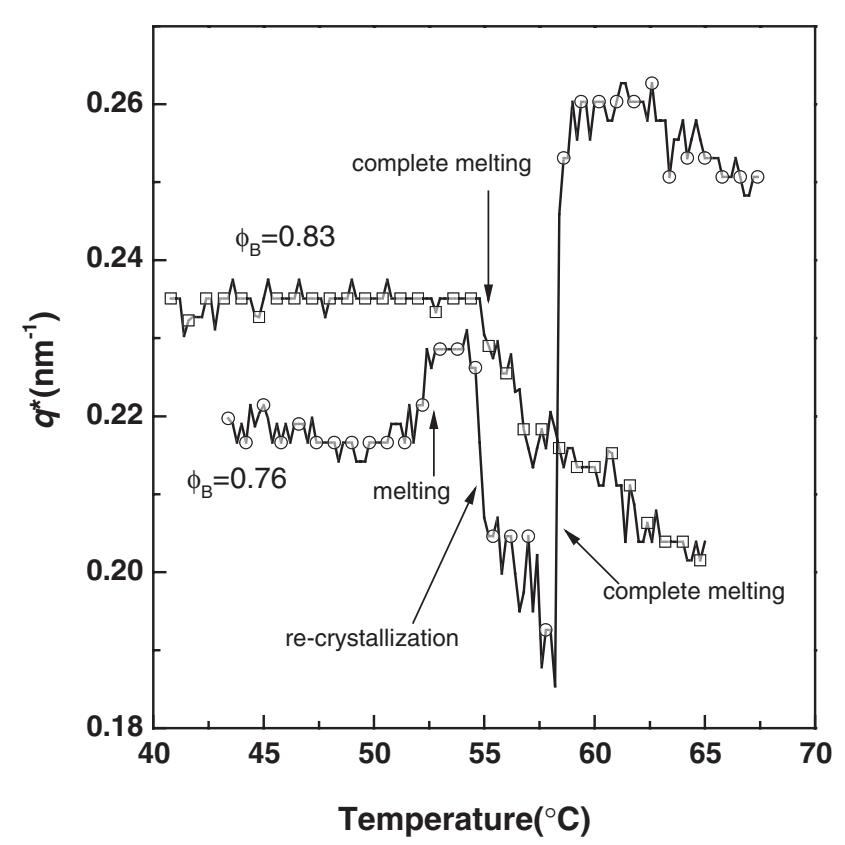

Figure 3. Variation in position of the first-order SAXS peak $\left(q^{*}\right)$ with temperature during melting. The heating rate is $0.5^{\circ} \mathrm{C} /$ min, starting from $40^{\circ} \mathrm{C}$.

the melting temperature, resulting from the different temperature ranges for the existence of PEO solid and melt during crystallization and melting (large supercooling is required for confined crystallization) and different deformation abilities of PEO solid and melt. The dimension of the liquid domains is recovered immediately after melting (see Figure 12 in ref 11). However, if the crystals are confined in cylinder domains and no re-organization takes place during melting, only once stepwise increase in $q^{*}$ should appear and no decrease in $q^{*}$ can be observed. ${ }^{11}$ Figure 3 shows that there is a stepwise increase in $q^{*}$ at $52^{\circ} \mathrm{C}$ for E155-76 and there is no gradual decrease in $q^{*}$ before such an increase in $q^{*}$. This corresponds to the melting of the confined PEO crystals with low crystallinity. Nevertheless, this is followed by a sudden decrease in $q^{*}$, and then stepwise increase in $q^{*}$. Abrupt decrease and discontinuous change of $q^{*}$ are characteristics of breakout crystallization, ${ }^{10}$ indicating the formation of new unconfined crystals. The last stepwise increase in $q^{*}$ corresponds to the melting of the unconfined crystals. ${ }^{11}$ The profile of $q^{*}$ versus temperature clearly shows that melting-recrystallization takes place during melting of E155-76 when heated at an extremely slow rate. The confined PEO crystals of E155-76 are first partially melted, then recrystallize into unconfined crystals before complete melting. In contrast, PEO crystals in E155-83 cannot reorganize via melting-recrystallization, though DSC results show that there are also less perfect crystals in E155-83.
Using atomic force microscopy, Rottele observed that the PEO crystals of different degrees of perfection in the poly(butadiene)- $b$-poly(ethylene oxide) are formed in different spherical domains due to different supercooling. ${ }^{15}$ The reason for this is that the sphere domain is very small and comparable to that of a crystalline nucleus, thus crystallization is controlled by nucleation rate, leading to an Avrami exponent of 1.0. ${ }^{7,8,11}$ Since the crystals in the same sphere domains have the same crystallinity and melt simultaneously. Once the crystals in a domain are melted, recrystallization of can only be initiated by the nucleus formed by thermal fluctuation. However, because the fusion enthalpy of the PEO crystals in individual domains is several orders of magnitude larger than the energy of thermal fluctuation, the equilibrium between the molten and crystalline states is prohibited and thus melting-recrystallization for the crystals confined in sphere domains cannot be observed. Nucleation is thus the key step to determine whether meltingrecrystallization happens. For the same reason, such melting-recrystallization cannot happen in the confined nano-cylinder domains, if the crystals in the same domains have the same perfection. But, in fact, melting-recrystallization process is observed E15576 , as shown in Figure 3. Thus the PEO crystals varying in perfection can be accommodated in the same cylinder domains. The crystals of less perfection probably recrystallize after melting, because the crystals of higher perfection can act as nuclei. This deduction is in accordance with isothermal kinetics. ${ }^{11}$ Avrami exponents for the blends with cylinder morphology systematically deviate from the typical Avrami exponent for confined crystallization controlled by the rate of homogeneous nucleation, which is 1.0. ${ }^{1,7,8,11,16}$ Diffusion may thus play a role in crystallization. ${ }^{17}$ Because of the high supercooling for confined crystallization and the large dimension in the cylinder direction, the crystallization rate is very slow in the cylinder direction, thus crystallization of the PEO cylinder domains is completed in a long time span, in which crystals of different perfection may be formed due to different supercooling.

\section{CONCLUSIONS}

The present work shows that the reorganization of the less perfect crystals in an environment depends on the morphology of the crystalline domains. For spherical domains, the polymer crystals with different degrees of perfection are distributed in different domains and reorganization via melting-recrystallization is impossible because of the difficulty in nucleation. In combination with Rottele's results, this conclusion is applicable to weakly segregated and 
strongly segregated block copolymers. PEO crystals of different perfection may be accommodated in the same cylinder domains due to the large dimension in the cylinder direction. Reorganization of less perfect crystals thus proceeds via melting-recrystallization since the crystals with higher perfection act as nuclei.

Acknowledgment. The authors thank Prof. A. J. Ryan at The University of Sheffield, U.K., for providing beamtime. This project was supported by National Natural Science Foundation of China (20374046) and by the Excellent Young Teachers Program of MOE, P. R. C.

\section{REFERENCES}

1. H. L. Chen, J. C. Wu, T. L. Lin, and J. S. Lin, Macromolecules, 34, 6936 (2001).

2. S. Nojima, M. Toei, S. Hara, S. Tanimoto, and S. Sasaki, Polymer, 43, 4087 (2002).

3. L. Zhu, Y. Chen, A. Q. Zhang, B. H. Calhoun, M. S. Chun, R. P. Quirk, S. Z. D. Cheng, B. S. Hsiao, F. J. Yeh, and T. Hashimoto, Phys. Rev. B, 60, 10022 (1999).

4. L. Zhu, S. Z. D. Cheng, B. H. Calhoun, Q. Ge, R. P. Quirk, E. L. Thomas, B. S. Hsiao, F. J. Yeh, and B. Lotz, J. Am. Chem. Soc., 122, 5957 (2000).

5. L. Zhu, B. R. Mimnaugh, Q. Ge, R. P. Quirk, S. Z. D.
Cheng, E. L. Thomas, B. Lotz, B. S. Hsiao, F. Yeh, and L. Z. Liu, Polymer, 42, 9121 (2001).

6. L. Zhu, S. Z. D. Cheng, B. H. Calhoun, Q. Ge, R. P. Quirk, E. L. Thomas, B. S. Hsiao, F. Yeh, and B. Lotz, Polymer, 42, 5829 (2001).

7. Y. L. Loo, R. A. Register, and A. J. Ryan, Phys. Rev. Lett., 84, 4120 (2000).

8. Y. L. Loo, R. A. Register, A. J. Ryan, and G. T. Dee, Macromolecules, 34, 8968 (2001).

9. Y. L. Loo, R. A. Register, and A. J. Ryan, Macromolecules, 35, 2365 (2002).

10. J. T. Xu, S. C. Turner, J. P. A. Fairclough, S. M. Mai, A. J. Ryan, C. Chaibundit, and C. Booth, Macromolecules, 35, 3614 (2002).

11. J. T. Xu, J. P. A. Fairclough, S. M. Mai, A. J. Ryan, and C. Chaibundit, Macromolecules, 35, 6937 (2002).

12. H. L. Chen, S. C. Hsiao, T. L. Lin, K. Yamauchi, H. Hasegawa, and T. Hashimoto, Macromolecules, 34, 671 (2001).

13. A. Rottele, T. Thurn-Albrecht, J. U. Sommer, and G. Reiter, Macromolecules, 36, 1257 (2003).

14. Y. Y. Huang, H. L. Chen, H. C. Li, T. L. Lin, and J. S. Lin, Macromolecules, 36, 282 (2003).

15. G. Reiter, G. Castelein, J. U. Sommer, A. Rottele, and T. Thurn-Albrecht, Phys. Rev. Lett., 87, 226101 (2001).

16. J. T. Xu, J. J. Yuan, and S. Y. Cheng, Eur. Polym. J., 39, 2091 (2003).

17. J. T. Xu, A. J. Ryan, S. M. Mai, J. J. Yuan, and S. Y. Cheng, J. Macromol. Sci., Part B: Phys., 43, 685 (2004). 